\title{
Internal leakage of oxygen flush valve
}

\author{
Sung Ha Mun, and Min Young No \\ Department of Anesthesiology and Pain Medicine, Kangbuk Samsung Hospital, Sungkyunkwan University School of Medicine, Seoul, \\ Korea
}

The oxygen flush valve receives oxygen from the pipeline inlet or cylinder pressure regulator and delivers a high flow of unmetered oxygen directly to the common gas outlet [1]. Internal leakage in the flush valve may result in delivery of an oxygen-enriched mixture to an anesthetized patient, causing lighter anesthesia than intended $[2,3]$. Authors report an experience of internal leakage in the oxygen flush valve owing to the wear of the O-ring.

A 6-year-old boy (height: $110 \mathrm{~cm}$, weight: $18 \mathrm{~kg}$ ) was admitted to the hospital for tonsillectomy. He had no particular medical history and no present illness categorized as American Society of Anesthesiologists class I. There were no abnormal findings on the pre-operative blood test, EKG, or the chest x-ray. Vital signs before induction of anesthesia were blood pressure 100/65 mmHg, heart rate (HR) 110 beats/min, and oxygen saturation $\left(\mathrm{SpO}_{2}\right) 99 \%$. Before anesthetic induction, a positivepressure leak test did not detect any leak. To induce anesthesia, thiopental sodium $100 \mathrm{mg}$ and rocuronium bromide $15 \mathrm{mg}$ were intravenously administered and endotracheal intubation was performed. Intraoperative anesthesia was maintained with sevoflurane 2-2.5 vol\%, $\mathrm{N}_{2} \mathrm{O} 2 \mathrm{~L} / \mathrm{min}$, and $\mathrm{O}_{2} 2 \mathrm{~L} / \mathrm{min}$ using the semiclosed-circuit anesthesia machine (Modulus ${ }^{\circledR} \mathrm{CD}$, DatexOhmeda, GE Healthcare, Madison, WI, USA). Intraoperative vital signs of the patient were systolic blood pressure 110-130 $\mathrm{mmHg}$, diastolic blood pressure 65-80 mmHg, HR 120-140 beats/ min, and $\mathrm{SpO}_{2} 100 \%$, showing no significant changes. While monitoring the child, however, an anesthesiologist found that the concentration of sevoflurane indicated on the anesthesia gas analyzer was significantly lower than that set at the sevoflurane vaporizer (the concentration at the sevoflurane vaporizer setting was $2 \%$ but at the gas analyzer was $1.2 \%$ ). In addition, the oxygen analyzer indicated that oxygen concentration was higher than the setting: oxygen was delivered at $50 \%$, but the oxygen concentration measurer indicated the delivery of $60 \%$ oxygen). Suspecting a malfunction of the vaporizer, at first, we checked whether or not the sevoflurane vaporizer was properly set up, but could not detect any particular problems. Then, we attempted desflurane for the inhaled anesthetics, but the issue was not resolved. In case the anesthetic depth may be lower than intended, fentanyl $25 \mu \mathrm{g}$ was intravenously provided. Because the operation was close to completion, we proceeded with the operation, while the sevoflurane vaporizer setting increased to $3 \mathrm{vol} \%$. After completion of the operation, we requested a repair on the machine to the manufacturer, and they discovered a leak in the oxygen flush valve. At the followup visit, following anesthesia, no awareness during general anesthesia was observed and he was discharged without any complications.

The oxygen flush valve is a device to allow direct communication between the oxygen high-pressure circuit and the lowpressure circuit. When the oxygen flush button is depressed, the oxygen flush valve will open and deliver $100 \%$ oxygen flow of 35 to $75 \mathrm{~L} / \mathrm{min}$ to the breathing circuit and mainly provide jet ventilation. Malfunction of the oxygen flush valve, although rare, may result in serious adverse events such as barotraumas or intra-anesthetic awareness. Mann et al. [3] reported an event of malfunction regarding the oxygen flush valve, where routine external checks revealed no problems and the valve superficially appeared to be normal. According to the report, a spiral fracture on the plastic shaft of the oxygen flush valve

Corresponding author: Sung Ha Mun, M.D., Department of Anesthesiology and Pain Medicine, Kangbuk Samsung Hospital, Sungkyunkwan University School of Medicine, 108, Pyoung-dong, Jongro-gu, Seoul 110-746, Korea. Tel: 82-2-2001-2001, Fax: 82-2-2001-2326, E-mail: anesmun@hanmail.net

(c) This is an open-access article distributed under the terms of the Creative Commons Attribution Non-Commercial License (http:// creativecommons.org/licenses/by-nc/3.0/), which permits unrestricted non-commercial use, distribution, and reproduction in any medium, provided the original work is properly cited. 


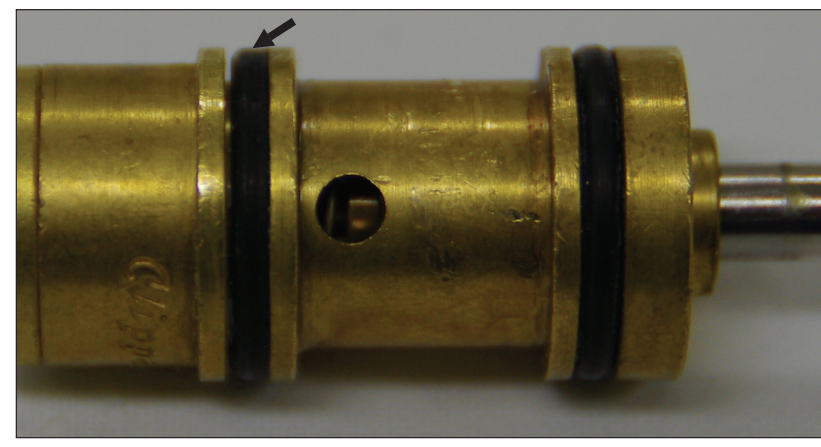

Fig. 1. An enlarged mechanical part of oxygen flush valve. We found the rubber of O-ring was worn out (arrow).

caused the flush valve to remain partially open, which allowed an additional flow of oxygen, approximately $4 \mathrm{~L} / \mathrm{min}$, into the breathing circuit and directed an oxygen-enriched mixture to the patient. Moreover, McMahon et al. [4] reported a case where gas flow from the flowmeter was cut off due to a defective oxygen flush valve.

Determined to discard it, we disassembled the unit. As a result, as shown in Fig. 1, we discovered that the O-ring around the oxygen flush valve was worn out and that oxygen seemed to have been leaking in as a result. In other words, the oxygen flush valve continued to be partially activated. Then, it is supposed that the continuous inflow of oxygen may have diluted the inhaled anesthetic agent, lowering the level of concentration more than expected. Furthermore, the oxygen concentration indicated on the oxygen analyzer seems to have been assessed much higher than the setting.

Pre-use leak tests to detect leakage in the anesthesia unit are performed daily in the operation room. The tests include the positive-pressure leak test and the negative-pressure leak test. For old models of anesthesia machines, the positive-pressure test is more commonly used because of its convenience. For new models of anesthesia machines, however, the test is inappropriate due to the presence of a check valve in the lowpressure circuit. On the other hand, the negative-pressure leak test can be applied to most anesthesia machines currently being used, regardless of the presence of the check valve, so it is called the universal leak test. Since the Ohmeda Modulus ${ }^{\circledR}$ $\mathrm{CD}$ machine used in the present case is an old model without a check valve, we conducted the positive pressure leak test. However, had we performed the negative-pressure leak test, we could have discovered the presence of a leak at least, even if unable to identify the exact location.

Even though most anesthesia machines undergo periodic inspections, such routine check-ups do not always discover all leak sites in an anesthesia machine. Therefore, a manual checkout procedure should be mandatory for thorough inspection of the machine. Prior to initiating anesthesia, it is required to perform daily pre-use leak tests using the checklists for anesthesia equipment issued in 1993 by the United States Food and Drug Administration [1].

An anesthesiologist should not dismiss any slight defects in the anesthesia machine because dealing with the unit is directly connected to a matter of life and death. Although the advancement in patient-monitoring skills and the automated check function of the anesthesia machine itself helps prevent many problems, it is necessary to be well-informed regarding suggestions on the pre-use inspections of anesthesia equipment and to have the anesthesia machine regularly checked by the biomedical engineering office.

\section{References}

1. Dorsch JA, Dorsch SE. Anesthesia machines and breathing systems. Equipment checkout and maintenance. In: Understanding anesthesia equipment. 5th ed. Philadelphia, Lippincott Williams \& Wilkins. 2008, p 100, 933.

2. Anonymous. Internal leakage from anesthesia unit flush valves. Health Devices 1981; 10: 7.

3. Mann DP, Der Ananian J, Alston TA. Oxygen flush valve booby trap. Anesthesiology 2004; 101: 558.

4. McMahon DJ, Holm R, Batra MS. Yet another machine fault. Anesthesiology 1983; 58: 586-7. 